\title{
Analysis of clinicopathological characteristics associated with the outcome of oral squamous cell carcinoma and the establishment of tissue microarrays
}

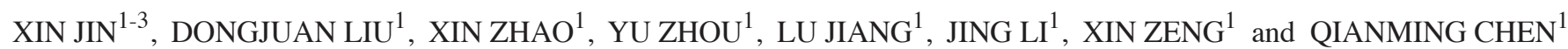 \\ ${ }^{1}$ State Key Laboratory of Oral Diseases, West China Hospital of Stomatology, Sichuan University, Chengdu, \\ Sichuan 610041; ${ }^{2}$ College of Stomatology, Chongqing Medical University, Chongqing 400016; \\ ${ }^{3}$ Chongqing Key Laboratory of Oral Diseases and Biomedical Sciences, Chongqing 401147, P.R. China
}

Received April 8, 2015; Accepted August 12, 2016

DOI: $10.3892 / \mathrm{ol} .2016 .5120$

\begin{abstract}
The aim of this study was to evaluate clinicopathological risk factors associated with the fatal outcome of oral squamous cell carcinoma (OSCC) in a large cohort of Chinese patients, and to construct tissue microarrays (TMAs) using this cohort. Univariate and multiple logistic regression analyses were performed to evaluate the predictors of poor prognosis in a cohort of 232 patients with OSCC, after which the patient tumor tissues were used to construct TMAs. Univariate logistic regression analysis indicated that a poor outcome of OSCC was associated with the male gender, a history of smoking, the tumor-node-metastasis stage and lymph node metastasis. Multiple logistic regression analysis demonstrated that an increased risk of mortality in patients with OSCC was significantly and independently associated with lymph node metastasis (odds ratio, 3.421; 95\% confidence interval, 1.609-7.273). Therefore, the results of the present study suggested that lymph node metastasis is an independent risk factor associated with a poor prognosis of OSCC patients. TMAs of OSCC were successfully constructed, and are the first TMAs to be reported in mainland Chinese patients.
\end{abstract}

\section{Introduction}

Oral squamous cell carcinoma (OSCC) is the sixth most common malignancy worldwide, accounting for $\sim 90 \%$ of malignant oral lesions (1). Despite improvements in its clinical management, OSCC continues to have a high local recurrence and a poor 5-year survival rate $(2,3)$. Local tumor recurrence

Correspondence to: Dr Qianming Chen or Dr Xin Zeng, State Key Laboratory of Oral Diseases, West China Hospital of Stomatology, Sichuan University, 14 Renmin Nanlu, Section 3, Chengdu, Sichuan 610041, P.R. China

E-mail: qmchen@scu.edu.cn

E-mail: zengxin22@163.com

Key words: oral squamous cell carcinoma, cohort study, tissue microarrays, lymph node metastasis develops in $\sim 60 \%$ of patients, and metastases affect $15-25 \%$, leading to a poor prognosis and encouraging further research on factors that might correlate with disease outcome (4).

At present, the tumor-node-metastasis (TNM) staging system is described as the most effective prognostic tool for tumor survival $(5,6)$. Furthermore, the clinical characteristics of patients, including their age, gender and smoking and drinking habits, are important for selecting the appropriate therapeutic strategy, and for determining the risk of complications and the prognosis of several types of cancer $(7,8)$. Therefore, the identification of factors associated with a poor prognosis has emerged as an important issue in the management of OSCC. Previous studies have evaluated various clinicopathological parameters as prognostic factors of OSCC, and the age (7) and smoking status (8) of patients, extracapsular spread in the cervical lymph nodes (9), TNM stage $(5,10)$, status of the surgical resection margin, bone involvement and the size of the mandibulectomy (11), as well as the tumor size and microvascular invasion $(12,13)$, have been shown to be independent prognostic factors in patients with OSCC. However, previous studies have assessed only few or groups of risk factors that might influence the prognosis, and the association between these factors and the prognosis of OSCC is inconsistent and complex. Therefore, it is important to identify characteristics that may be suggestive of a poor prognosis in patients with OSCC.

The construction of tissue microarrays (TMAs), in which multiple samples are incorporated into a single paraffin block, allows for the immunohistochemical analysis of large numbers of tissue samples simultaneously, thereby standardizing immunohistochemical staining (14). Not only does this markedly reduce costs, but it also increases the number of studies that can be performed on a small piece of tissue, since smaller cores of tissue are used rather than cut sections (15). By using TMAs, Lourenço et al (16) demonstrated that claudin expression patterns were related to the evolution and prognosis of OSCC. Furthermore, Liu et al (17) reported that the expression levels of carbonic anhydrases (CAs) I/II in OSCC samples may be used to predict local tumor growth in OSCC patients. However, there has yet to be a comparable analysis of OSCC patients from the mainland Chinese population. 
The present study has taken into consideration numerous characteristics and prognostic factors, in order to identify important independent risk factors of a poor prognosis in a relatively large cohort of Chinese patients with OSCC. In addition, this cohort of patients was used to construct the first OSCC TMAs from mainland Chinese patients.

\section{Patients and methods}

Study participants. After obtaining approval from the West China Hospital Ethics Board (Chengdu, China), a cohort of 232 histologically confirmed OSCC patients was recruited from the West China Hospital of Stomatology, Sichuan University (Chengdu, China) between February 2002 and October 2009. Patients were eligible for data collection if they met the following inclusion criteria: i) They had been histologically diagnosed with oral squamous cell carcinoma; and ii) they had been treated with primary surgery. The exclusion criteria were: i) Recurrent or metastatic disease; and ii) malignant disease of the salivary glands, tonsils and oropharynx or hypopharynx.

Data collection. Clinicopathological parameters were obtained by a registry review, and included: i) Demographic data, such as age, gender and history of smoking and alcohol consumption; ii) tumor-related information, including location, size, TNM stage [the patients were staged according to the American Joint Committee on Cancer TNM classification system (18)], histological differentiation and lymphatic metastasis; and iii) status at the last follow-up (April 30, 2011), mainly the condition of survival or mortality.

Statistical analysis. Categorical variables are expressed as frequencies and percentages. To compare the data between two groups, the Pearson $\chi^{2}$ test was implemented for qualitative variables. Univariate logistic regression $\left(\chi^{2}\right.$ test with Yate's continuity correction) was performed for the demographic and clinical characteristic variables that were significantly associated with an increased risk of mortality. Factors with $\mathrm{P}<0.25$ in the univariate analysis were included for multivariate logistic regression analysis to identify the independency of these factors (the $\alpha$-level of significance was relaxed to 0.25 in order to avoid missing important variables). Independent variables with $\mathrm{P}<0.05$ in the multiple logistic regression analysis were selected for receiver operating characteristic (ROC) curve analysis. Plots of sensitivity (true-positive fraction) vs. 1-specificity (false-positive fraction) were constructed, and the diagnostic performance of the significant independent variable was expressed as the area under the ROC curve (AUC). All statistical analyses were performed using SPSS 17.0 software (SPSS Inc., Chicago, IL, USA). For unadjusted comparisons, $\mathrm{P}<0.05$ was considered to indicate a statistically significant difference.

TMAs design and construction. According to the characteristics of OSCC donor paraffin wax (OSCC tissues with or without lymph node metastasis, the pericarcinomatous tissues or the metastatic lymph node tissues that met the inclusion criteria), we designed four sets of arrays, the arrangements of which were as follows: i) TMA1, which included 162 tissue cores from 81 patients (Fig. 1A); ii) TMA2, which included 118 tissue cores from 58 patients (Fig. 1B); iii) TMA3, which included 161 tissue cores from 161 patients (Fig. 1C); and iv) TMA4, which included 162 tissue cores from 162 patients (Fig. 1D).

The representative pathology regions were observed by hematoxylin and eosin (HE) staining, after which the HE-stained sections were overlaid onto the surface of the corresponding donor blocks to guide the transfer of tissue cores from morphologically representative sites into defined loci in the recipient block using a steel stylet. Tissue cores with a diameter of $1.5 \mathrm{~mm}$, and the recipient blocks, were heated in a $52^{\circ} \mathrm{C}$ oven until they had fused to become the TMA blocks. Consecutive sections (4- $\mu \mathrm{m}$ thick) were cut, and every 10 slides were collected to assess by HE staining. The specific processes were performed as described previously (14).

\section{Results}

Clinical characteristics. Clinicopathological parameters of 232 patients with OSCC, including 175 fatal cases and 57 non-fatal cases, were analyzed. The 232 patients with OSCC included 169 males and 63 females, with an age range of 24-83 years. The 57 non-fatal cases included 37 males and 20 females, of which 26 patients were aged $>60$ years. Of the 175 fatal cases, 132 were male and 43 were female, and 82 patients were aged $>60$ years. There was no significant difference in age and gender between the non-fatal and fatal groups $(\mathrm{P}=0.918$ and 0.121 , respectively; Table I). Similarly, no significant differences in smoking and alcohol consumption were observed between the fatal and non-fatal groups (smoking habit, 53.1 vs. 43.9\%, respectively; alcohol consumption, 50.9 vs. $50.9 \%$, respectively; $\mathrm{P}=0.223$ and 0.998 , respectively). The baseline clinical presentations were similar between the fatal and non-fatal groups in terms of the tumor stage, size and location ( $\mathrm{P}=0.117,0.799$ and 0.972 , respectively). However, lymphatic metastasis was significantly more frequent in fatal patients than in non-fatal patients ( 78.3 vs. $17.5 \%$, respectively; $\mathrm{P}=0.002$; Table I). In addition, well-differentiated OSCC was significantly associated with fatal cases $(\mathrm{P}=0.049)$. The basic characteristics of the 232 study participants are shown in Table I.

Determinants of a poor OSCC prognosis. The associations between the clinical characteristics of patients with OSCC and a poor prognosis were evaluated. Univariate logistic regression analysis revealed that the male gender $(\mathrm{P}=0.123)$, a history of smoking $(\mathrm{P}=0.225)$, tumor stages I and III $(\mathrm{P}=0.129$ and 0.147 , respectively), and lymphatic metastasis $(\mathrm{P}=0.002)$ were significantly associated with patient survival (Table II).

Table III shows the final model for the predictors in the multivariate logistic regression analysis to identify factors that were independently associated with a poor prognosis. The risk of mortality in patients with lymphatic metastasis was 3.421-times higher than those with normal lymph nodes, after adjusting for gender, smoking history and TNM stage (95\% confidence interval, 1.609-7.273; $\mathrm{P}=0.001$ ), which was demonstrated as independent prognostic factor in all OSCCs. The male gender $(\mathrm{P}=0.077)$, a history of smoking $(\mathrm{P}=0.438)$ and the TNM stage $(\mathrm{P}>0.05)$ were not shown to be independent prognostic factors of OSCC. The AUC for the prediction model was 0.62 (Fig. 2). 
A

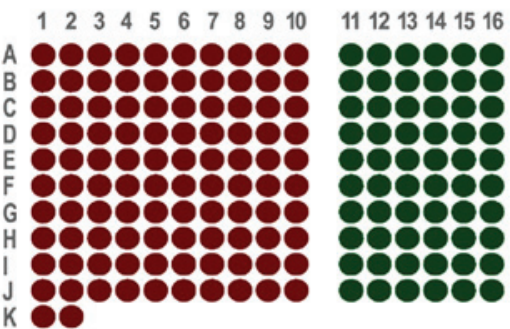

C

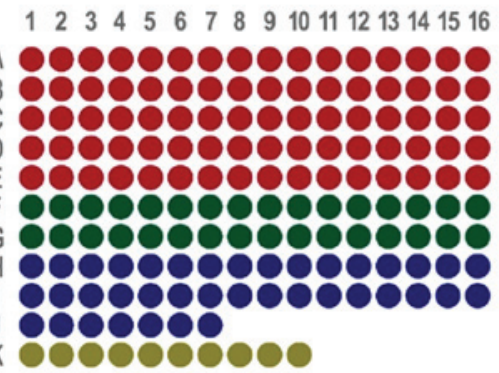

B
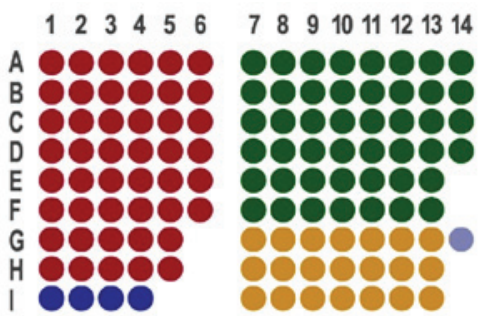

D

12345678910111213141516

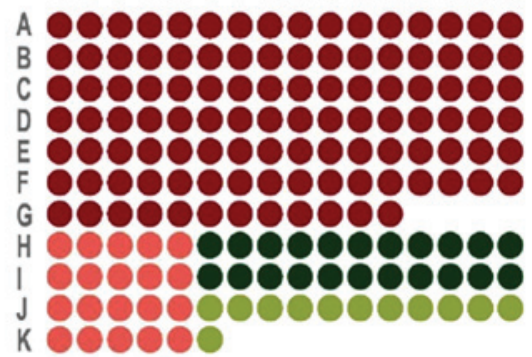

Figure 1. Design and construction of tissue microarrays (TMAs) using tissue cores from 232 patients with oral squamous cell carcinoma (OSCC) from mainland China. (A) Arrangement of tissue cores in TMA1. The red dots indicate cancerous tissues without lymph node metastasis and pericarcinomatous tissues (51 samples). The green dots indicate cancerous tissues with lymph node metastasis and pericarcinomatous tissues (30 samples). (B) Arrangement of tissue cores in TMA2. The red dots represent cancerous tissues without lymph node metastasis and pericarcinomatous tissues (23 samples). The green dots represent cancerous tissues with lymph node metastasis and pericarcinomatous tissues ( 23 samples). The blue dots correspond to cancerous tissues without lymph node metastasis and pericarcinomatous tissues within the same dot (4 samples). The yellow dots indicate cancerous tissues with lymph node metastasis, pericarcinomatous tissues and a metastatic lymph node located in the corresponding 3 cores ( 7 samples). The grey/purple dots correspond to cancerous tissues with lymph node metastasis and pericarcinomatous tissues in the same loci (1 sample). (C) Arrangement of tissue cores in TMA3. The red dots correspond to cancerous tissues without lymph node metastasis ( 80 samples). The green dots represent cancerous tissues with lymph node metastasis (32 samples). The blue dots correspond to metastatic lymph node tissues (39 samples). The yellow dots represent non-metastatic lymph node tissues (10 samples). (D) Arrangement of tissue cores in TMA4. The red dots indicate cancerous tissues without lymph node metastasis (108 samples). The pink dots correspond to pericarcinomatous tissues without lymph node metastasis (20 samples). The green dots indicate cancerous tissues with lymph node metastasis ( 22 samples). The yellow dots represent metastatic lymph node tissues (12 samples).

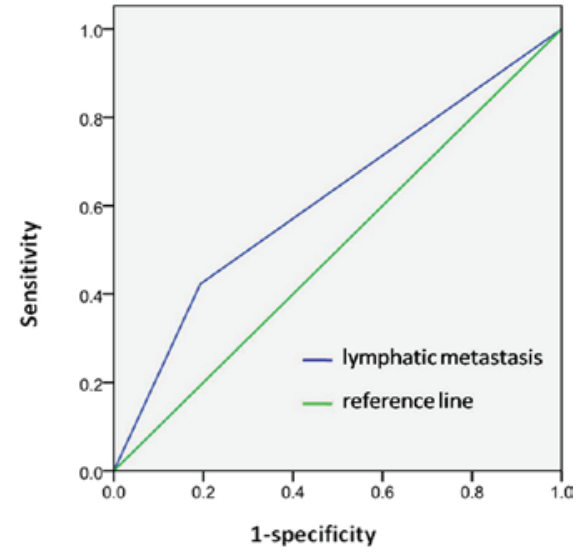

Figure 2. Receiver operating characteristics curve. Model was obtained from the multivariate logistic regression with the area under the curve $=0.62$.

HE staining of TMAs. HE staining of the four TMAs is shown in Fig. 3. HE staining identified cancerous tissues (Fig. 4A), pericarcinomatous tissues (Fig. 4B) and metastatic lymph node tissues (Fig. 4C).

\section{Discussion}

A previous study suggested that cohort studies could be employed to support the existence of associations between suspected risk factors and disease outcome (19). OSCC accounts for $\sim 90 \%$ of malignant oral lesions, and is widely recognized as the most frequently occurring malignant tumor of oral structures (2). The mortality rate of OSCC is relatively high, with a 5-year survival rate of $50 \%$ (20). Therefore, the present study analyzed clinical symptoms and pathological parameters in 232 cases of OSCC, including 175 that were fatal and 57 that were non-fatal, in order to identify factors associated with a poor prognosis. The parameters of gender, age, smoking and drinking habits, tumor location, size and stage, as well as lymphatic metastasis, were analyzed. Notably, a multiple regression analysis identified lymphatic metastasis as an important independent factor associated with a fatal outcome of OSCC. To the best of our knowledge, this is one of the largest studies to assess numerous factors as survival predictors in patients with OSCC.

Previous reports on risk factors and a poor prognosis of OSCC have indicated the existence of various and inconsistent determinants (7,12). Grimm (12) retrospectively reviewed pathological parameters of patients with OSCC, including age, gender, site distribution, tumor size, lymph node involvement and grading, and demonstrated that an increased tumor size and microvascular invasion were independent prognostic factors for predicting survival in patients with OSCC. However, Rossi et al (7) reported that patients diagnosed before reaching 65 years-of-age showed an improved prognosis, as compared with patients diagnosed when aged $>65$ years, while the gender distribution was almost equal. 
Table I. Clinical characteristics of fatal and non-fatal oral squamous cell carcinoma cases.

\begin{tabular}{|c|c|c|c|}
\hline Variable & Fatal $(n=175)$ & Non-fatal $(n=57)$ & P-value \\
\hline Gender ( $\%$ male $)$ & $132(75.4)$ & $37(64.9)$ & 0.121 \\
\hline Age (years) & & & 0.918 \\
\hline$<40$ & $14(8.1)$ & $4(7.0)$ & \\
\hline $40-60$ & $77(44.5)$ & $27(47.4)$ & \\
\hline$>60$ & $82(47.4)$ & $26(45.6)$ & \\
\hline Smoking & $93(53.1)$ & $25(43.9)$ & 0.223 \\
\hline Alcohol consumption & $89(50.9)$ & $29(50.9)$ & 0.998 \\
\hline Location & & & 0.972 \\
\hline Buccal mucosa & $25(14.3)$ & $7(12.3)$ & \\
\hline Tongue & $48(27.4)$ & $17(29.8)$ & \\
\hline Gingiva & $26(14.9)$ & $8(14.0)$ & \\
\hline Other sites & $76(43.4)$ & 25 (43.9) & \\
\hline Tumor size (cm) & & & 0.799 \\
\hline$<2$ & $54(33.5)$ & $16(29.6)$ & \\
\hline $2-4$ & $84(52.2)$ & $31(57.4)$ & \\
\hline$\geq 4$ & $23(14.3)$ & $7(13.0)$ & \\
\hline Differentiated type & & & $0.049^{\mathrm{a}}$ \\
\hline Well & $158(91.3)$ & $55(98.2)$ & \\
\hline Moderately & $10(5.8)$ & $0(0.0)$ & \\
\hline Poorly & $5(2.9)$ & $1(1.8)$ & \\
\hline TNM stage & & & 0.117 \\
\hline $\mathrm{I}$ & $27(15.9)$ & $10(18.2)$ & \\
\hline II & $34(20.0)$ & $18(32.7)$ & \\
\hline III & $42(24.7)$ & $7(12.7)$ & \\
\hline IV & $67(39.4)$ & $20(36.4)$ & \\
\hline Lymphatic metastasis & $137(78.3)$ & $10(17.5)$ & $0.002^{\mathrm{a}}$ \\
\hline
\end{tabular}

Values are presented as $\mathrm{n}(\%)$. Certain data are missing due to incomplete medical records or as teh patients were lost to follow-up. The Pearson $\chi^{2}$ test was used to compare qualitative variables. Statistical analyses were performed using SPSS 17.0 software. ${ }^{\text {a }}<0.05$ was considered statistically significant. TNM, tumor-node-metastasis.

The TNM staging system has been widely accepted as the basis of cancer staging, since it provides a reasonably precise description of the extent of disease (21). The main failing of the system, however, is that it does not include information about the clinical features of the patient. Ribeiro et al (5) demonstrated that survival estimates may be improved by the addition of patient clinical features to the TNM staging system, generating a more powerful and precise system in the determination of prognosis.

Kang et al (22) reported that the presence of pathological node metastases was an independent predictor of the 5 -year outcomes of 467 patients, although, only for those patients with well-differentiated OSCC. Kawakita et al (8) conducted a retrospective cohort analysis of 222 patients with OSCC, in which they assessed the association between smoking status of patients and clinical outcome. Furthermore, Munoz Guerra et al (11) analyzed a 20-year cohort of 106 patients to assess the association between a worse prognosis and factors related to the surgical treatment of oral cancer.
No definitive assumptions regarding the prognostic factors for OSCC outcome can be drawn, due to the incomplete design or small sample size of previous studies (15-17). Therefore, the present study aimed to screen as many factors as possible, including age, gender, history of smoking and alcohol consumption, tumor location and size, TNM stage, histological differentiation and lymphatic metastasis, in a relatively large cohort, in order to obtain a more precise estimation of the independent predictors for a worse prognosis. Univariate logistic regression analysis revealed that the smoking status, male gender, tumor stages I and III, and lymphatic metastasis were significantly associated with clinical outcomes. However, following a multivariate logistic regression analysis, only lymph node metastasis was identified as an independent risk factor for a poor prognosis.

An association between smoking and the risk of developing OSCC has previously been demonstrated $(23,24)$; however, the relationship between the smoking status and clinical outcome of a patient remains inconclusive. Previous studies have reported that tobacco smoke promotes tumor hypoxia associated with 
Table II. Univariate logistic regression analysis.

\begin{tabular}{|c|c|c|c|c|}
\hline Variable & $\mathrm{B}$ & SE & P-value & OR $(95 \% \mathrm{CI})$ \\
\hline Gender (male) & -0.506 & 0.328 & $0.123^{\mathrm{a}}$ & $0.603(0.317-1.147)$ \\
\hline \multicolumn{5}{|l|}{ Age (years) } \\
\hline$<40$ & 0.104 & 0.610 & 0.864 & $1.110(0.336-3.668)$ \\
\hline $40-60$ & -0.101 & 0.317 & 0.751 & $0.904(0.486-1.684)$ \\
\hline$>60^{\mathrm{b}}$ & & & 0.919 & \\
\hline Smoking & 0.373 & 0.307 & $0.225^{\mathrm{a}}$ & $1.452(0.795-2.649)$ \\
\hline Alcohol consumption & 0.000 & 0.305 & 0.998 & $0.999(0.550-1.817)$ \\
\hline \multicolumn{5}{|l|}{ Location } \\
\hline Buccal mucosa & 0.161 & 0.486 & 0.740 & $1.175(0.453-3.044)$ \\
\hline Tongue & -0.074 & 0.364 & 0.839 & $0.929(0.455-1.897)$ \\
\hline Gingiva & 0.067 & 0.465 & 0.886 & $1.069(0.429-2.662)$ \\
\hline Other sites $^{\mathrm{b}}$ & & & 0.972 & \\
\hline \multicolumn{5}{|l|}{ Tumor size (cm) } \\
\hline$<2^{b}$ & & & 0.800 & \\
\hline $2-4$ & -0.220 & 0.354 & 0.535 & $0.803(0.401-1.606)$ \\
\hline$\geq 4$ & -0.027 & 0.517 & 0.959 & $0.974(0.353-2.682)$ \\
\hline \multicolumn{5}{|l|}{ Differentiated type } \\
\hline Well $^{\mathrm{b}}$ & & & 0.882 & \\
\hline Moderately & 20.148 & $1.271 \times 10^{4}$ & 0.999 & $0.562(0.000-5.347)$ \\
\hline Poorly & 0.554 & 1.107 & 0.617 & $1.741(0.199-15.226)$ \\
\hline \multicolumn{5}{|l|}{ TNM } \\
\hline $\mathrm{I}^{\mathrm{b}}$ & & & $0.129^{\mathrm{a}}$ & \\
\hline II & -0.357 & 0.471 & 0.448 & $0.700(0.278-1.762)$ \\
\hline III & 0.799 & 0.551 & $0.147^{\mathrm{a}}$ & $2.222(0.755-6.545)$ \\
\hline IV & 0.216 & 0.449 & 0.631 & $1.241(0.514-2.994)$ \\
\hline Lymphatic metastasis & 1.120 & 0.369 & $0.002^{\mathrm{a}}$ & 3.064 (1.487-6.313) \\
\hline
\end{tabular}

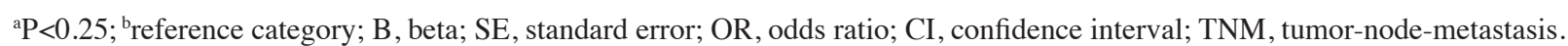

Table III. Multivariate logistic regression analysis.

\begin{tabular}{lcccc}
\hline Variable & $\mathrm{B}$ & $\mathrm{SE}$ & P-value & OR $(95 \% \mathrm{CI})$ \\
\hline Gender (male) & -0.607 & 0.343 & 0.077 & $0.545(0.278-1.067)$ \\
Smoking & 0.304 & 0.392 & 0.438 & $1.356(0.629-2.923)$ \\
TNM & & & & \\
I $^{\mathrm{a}}$ & & & 0.516 & \\
II & -0.357 & 0.483 & 0.460 & $0.700(0.271-1.804)$ \\
III & 0.392 & 0.573 & 0.494 & $1.480(0.481-4.550)$ \\
IV & -0.206 & 0.477 & 0.666 & $0.814(0.319-2.074)$ \\
Lymphatic & 1.230 & 0.385 & $0.001^{\mathrm{b}}$ & $3.421(1.609-7.273)$ \\
metastasis & & & &
\end{tabular}

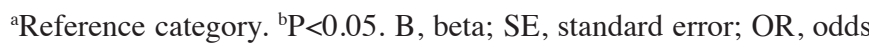
ratio; $\mathrm{CI}$, confidence interval; TNM, tumor-node-metastasis.

resistance to radiotherapy (RT) and upregulation of the epidermal growth factor receptor, and demonstrated that a mutation in the p53 gene was associated with resistance to apoptosis (25-27).
Kawakita et al (8) hypothesized that the pre-treatment smoking status of patients with OSCC was associated with survival, but only in patients receiving chemoradiotherapy (CRT)/RT, thus suggesting that the impact of smoking on patient survival may differ according to the treatment method. In addition, several studies have reported that smoking cessation during RT was beneficial to clinical outcome (28-30). In the present study, smoking was not considered an independent factor.

In the present study, the age and gender distributions of patients were not significantly different between the fatal and non-fatal cases, which was mainly consistent with previous reports $(1,7)$. Even when the incidence of OSCC has been shown to be age- or gender-related (31), it is difficult to consider them as independent prognostic factors.

OSCC often metastasizes to the lymph nodes, which was shown in the present study to be the most important predictor of patient survival. Approximately $50 \%$ of patients with OSCC have detectable lymph node involvement, and $<40 \%$ of patients with lymph node metastasis survive for 5 years, as compared with $90 \%$ of patients without metastasis (32). Therefore, lymph node metastasis may be considered important for predicting the prognosis of patients with OSCC. Generally, the 
A

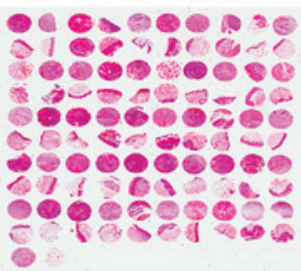

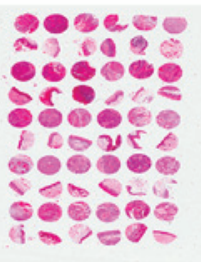

C

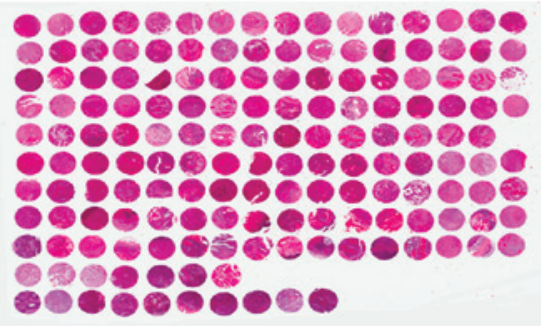

B

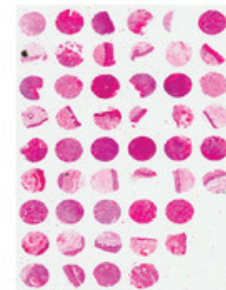

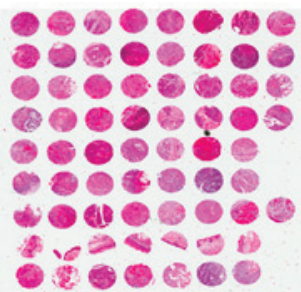

D

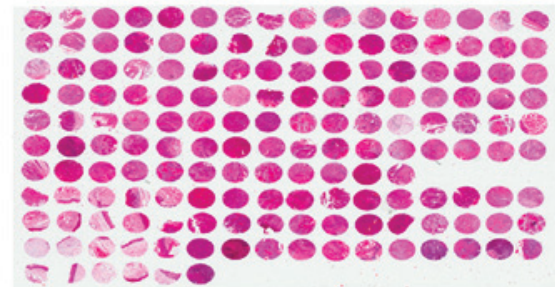

Figure 3. Hematoxylin and eosin staining of the four tissue microarrays (TMAs) constructed using tissue cores from 232 patients with oral squamous cell carcinoma (OSCC) from mainland China (A) TMA1, (B) TMA2, (C) TMA3 and (D) TMA4.
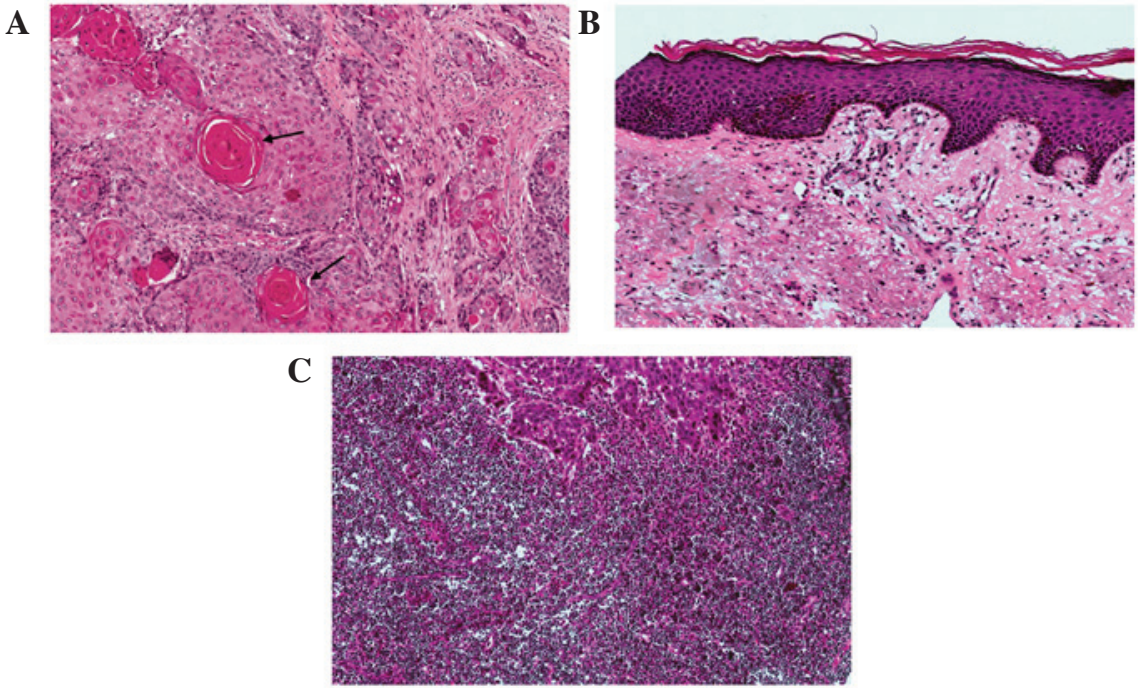

Figure 4. Hematoxylin and eosin staining of (A) cancerous tissues (black arrow indicate cancer nests), (B) pericarcinomatous tissues and (C) metastastic lymph node tissues. Original magnification, x100.

TNM staging system, which is used to describe the anatomical extension of the disease, is considered the most important prognostic factor $(5,6)$, which is inconsistent with the results of the present study. The TNM staging system consists of three parts (tumor, lymph node and metastasis), and it may be that the tumor size is not as important as lymphatic metastasis in determining the prognosis of a patient; thus, the tumor size may be a confounding factor when analyzing the TNM stage as a whole. For example, Kang et al (22) hypothesized that, in OSCC patients with pathologically negative node disease, a tumor depth of $\geq 8 \mathrm{~mm}$ (rather than tumor size) was the only independent prognostic factor for 5-year outcomes. However, the error caused by the small sample size for each stage of TNM system may be the reason for this result.

Increasingly, studies have focused on identifying the molecular markers associated with the diagnosis, treatment and prognosis of OSCC (33). The TMA is a high-throughput method that has been used due to its high efficiency, large-scale, ability to save time, small sample requirements, and standardization (14). To date, few previous studies have employed OSCC TMAs. Chen et al (34) used an OSCC TMA to demonstrate that the expression of the fascin protein may have an important role in the progression of OSCC, while Chien et al (35) determined that the expression of CA XII in OSCC samples could predict the progression of OSCC and survival of OSCC patients. In order to form an experimental platform for the further detection of markers associated with OSCC, the present study used the tumor tissues of 232 patients with OSCC to construct TMAs. Each chip contained the target tissue cores, including carcinoma tissues, pericancerous tissues and lymph nodes. The epidemiological and clinical data of the patients used for construction of the TMAs was completely recorded. To the best of our knowledge, these are the first OSCC TMAs of the mainland Chinese population to be constructed, and include a large sample size and detailed records. They will form the experimental base for the further exploration of the molecular targets associated with OSCC. 
The present study had some limitations. First, tumor differentiation has been shown to affect the treatment outcome in patients with OSCC, and poor differentiation typically indicates that the tumor cells have lost the characteristics of epithelial cells and have a more primitive nature $(36,37)$. Chen et al $(38)$ reported that a poorly-differentiated tumor was an independent risk factor for subsequent distant metastasis in patients with locoregionally advanced OSCC. However, the results of the present study suggested that tumor differentiation was not significantly associated with a poor outcome in a univariate logistic regression analysis. The reason for this could be that the number of patients with poorly- and moderately-differentiated tumors in the cohort was too small (10 moderately and 6 poorly), especially compared with the large number of patients with well-differentiated tumors (213 participants); this may have generated large standard errors and inaccurate regression coefficients. Second, this study overlooked some other factors, including comorbidities and treatment method, which may have affected the prognosis. Third, since the specific survival time of each patient was not obtained, a survival analysis could not be performed to estimate the time until death. Fourth, no definitive assumptions could be drawn due to the relatively small number of subjects in this retrospective cohort study. Therefore, a future OSCC cohort should include more participants, especially the current groups of small population.

In conclusion, the present study demonstrated that lymphatic metastasis was an independent risk factor for the prediction of a poor prognosis in a large OSCC patient cohort, in which a large number of clinicopathological variables were screened. However, more balanced cohort data are required to identify more precise prognostic factors. Furthermore, using this cohort, the first OSCC TMAs to be reported for a mainland Chinese population were successfully established.

\section{Acknowledgements}

The present work was supported by grants from the National Natural Science Foundation of China (grant nos. 81321002, 81472533 and 81500855), the International Science and Technology Cooperation Program of China (grant no. 2012DFA31370), the Chongqing Research Program of Basic Research and Frontier Technology (grant no. cstc2015jcyjA10029) and the Program for Innovation Team Building at the Institutions of Higher Education in Chongqing in 2016.

\section{References}

1. Warnakulasuriya S: Global epidemiology of oral and oropharyngeal cancer. Oral Oncol 45: 309-316, 2009.

2. Kamarajan P, Garcia-Pardo A, D'Silva NJ and Kapila YL: The CS1 segment of fibronectin is involved in human OSCC pathogenesis by mediating OSCC cell spreading, migration, and invasion. BMC Cancer 10: 330, 2010.

3. Wu CH, Wu TY, Li CC, Lui MT, Chang KW and Kao SY: Impact of diabetes mellitus on the prognosis of patients with oral squamous cell carcinoma: A retrospective cohort study. Ann Surg Oncol 17: 2175-2183, 2010.

4. Genden EM, Ferlito A, Bradley PJ, Rinaldo A and Scully C: Neck disease and distant metastases. Oral Oncol 39: 207-212, 2003.

5. Ribeiro KC, Kowalski LP and Latorre MR: Impact of comorbidity, symptoms, and patients' characteristics on the prognosis of oral carcinomas. Arch Otolaryngol Head Neck Surg 126: 1079-1085, 2000.
6. Fan S, Tang QL, Lin YJ, Chen WL, Li JS, Huang ZQ, Yang ZH, Wang YY, Zhang DM, Wang HJ, et al: A review of clinical and histological parameters associated with contralateral neck metastases in oral squamous cell carcinoma. Int J Oral Sci 3: 180-191, 2011.

7. Rossi V, Tarozzi M, Lodi G, Sardella A, Demarosi F and Carrassi A: Clinical aspect and survival rates in subject with oral cancer: A retrospective cohort study. Minerva Stomatol 56: 591-601, 2007 (In English, Italian).

8. Kawakita D, Hosono S, Ito H, Oze I, Watanabe M, Hanai N, Hasegawa Y, Tajima K, Murakami S, Tanaka H and Matsuo K: Impact of smoking status on clinical outcome in oral cavity cancer patients. Oral Oncol 48: 186-191, 2012.

9. Shaw RJ, Lowe D, Woolgar JA, Brown JS, Vaughan ED, Evans C, Lewis-Jones H, Hanlon R, Hall GL and Rogers SN: Extracapsular spread in oral squamous cell carcinoma. Head Neck 32: 714-722, 2010.

10. Kreppel M, Dreiseidler T, Rothamel D, Eich HT, Drebber U, Zöller JE and Scheer M: The role of clinical versus histopathological staging in patients with advanced oral squamous cell carcinoma treated with neoadjuvant radiochemotherapy followed by radical surgery. J Craniomaxillofac Surg 41: 22-27, 2013.

11. Muñoz Guerra MF, Naval Gías L, Campo FR and Pérez JS: Marginal and segmental mandibulectomy in patients with oral cancer: A statistical analysis of 106 cases. J Oral Maxillofac Surg 61: 1289-1296, 2003.

12. Grimm M: Prognostic value of clinicopathological parameters and outcome in 484 patients with oral squamous cell carcinoma: Microvascular invasion $\left(\mathrm{V}^{+}\right)$is an independent prognostic factor for OSCC. Clin Transl Oncol 14: 870-880, 2012.

13. Ratajczak-Wrona W, Jablonska E, Antonowicz B, Dziemianczyk D and Grabowska SZ: Levels of biological markers of nitric oxide in serum of patients with squamous cell carcinoma of the oral cavity. Int J Oral Sci 5: 141-145, 2013.

14. Liu CL, Prapong W, Natkunam Y, Alizadeh A, Montgomery K, Gilks CB and van de Rijn M: Software tools for high-throughput analysis and archiving of immunohistochemistry staining data obtained with tissue microarrays. Am J Pathol 161: 1557-1565, 2002.

15. Kononen J, Bubendorf L, Kallioniemi A, Bärlund M, Schraml P, Leighton S, Torhorst J, Mihatsch MJ, Sauter G and Kallioniemi OP: Tissue microarrays for high-throughput molecular profiling of tumor specimens. Nat Med 4: 844-847, 1998.

16. Lourenço SV, Coutinho-Camillo CM, Buim ME, Pereira CM, Carvalho AL, Kowalski LP and Soares FA: Oral squamous cell carcinoma: Status of tight junction claudins in the different histopathological patterns and relationship with clinical parameters. A tissue-microarray-based study of 136 cases. J Clin Pathol 63: 609-614, 2010

17. Liu CM, Lin YM, Yeh KT, Chen MK, Chang JH, Chen CJ, Chou MY, Yang SF and Chien MH: Expression of carbonic anhydrases I/II and the correlation to clinical aspects of oral squamous cell carcinoma analyzed using tissue microarray. J Oral Pathol Med 41: 533-539, 2012.

18. Edge SB and Compton CC: The American Joint Committee on Cancer: The 7th edition of the AJCC cancer staging manual and the future of TNM. Ann Surg Oncol 17: 1471-1474, 2010.

19. Doll R: Cohort studies: History of the method. II. Retrospective cohort studies. Soz Praventivmed 46: 152-160, 2001.

20. Wang Z, Jiang L, Huang C, Li Z, Chen L, Gou L, Chen P, Tong A, Tang M, Gao F, et al: Comparative proteomics approach to screening of potential diagnostic and therapeutic targets for oral squamous cell carcinoma. Mol Cell Proteomics 7: 1639-1650, 2008.

21. Schwab W: The present state of the guideline of the UICC on the TNM system. Arch Klin Exp Ohren Nasen Kehlkopfheilkd 191: 634-636, 1968 (In German).

22. Kang CJ, Liao CT, Hsueh C, Lee LY, Lin CY, Fan KH, Wang HM, Huang SF, Chen IH, Ng SH, et al: Outcome analysis of patients with well-differentiated oral cavity squamous cell carcinoma. Oral Oncol 47: 1085-1091, 2011.

23. Hashibe M, Brennan P, Benhamou S, Castellsague X, Chen C, Curado MP, Dal Maso L, Daudt AW, Fabianova E, Fernandez L, et al: Alcohol drinking in never users of tobacco, cigarette smoking in never drinkers, and the risk of head and neck cancer: Pooled analysis in the international head and neck cancer epidemiology consortium. J Natl Cancer Inst 99: 777-789, 2007.

24. La Vecchia C, Tavani A, Franceschi S, Levi F, Corrao G and Negri E: Epidemiology and prevention of oral cancer. Oral Oncol 33: 302-312, 1997. 
25. Liloglou T, Scholes AG, Spandidos DA, Vaughan ED, Jones AS and Field JK: p53 mutations in squamous cell carcinoma of the head and neck predominate in a subgroup of former and present smokers with a low frequency of genetic instability. Cancer Res 57: 4070-4074, 1997.

26. Jensen JA, Goodson WH, Hopf HW and Hunt TK: Cigarette smoking decreases tissue oxygen. Arch Surg 126: 1131-1134, 1991

27. Toyooka S, Matsuo K, Shigematsu H, Kosaka T, Tokumo M, Yatabe Y, Ichihara S, Inukai M, Suehisa H, Soh J, et al: The impact of sex and smoking status on the mutational spectrum of epidermal growth factor receptor gene in non small cell lung cancer. Clin Cancer Res 13: 5763-5768, 2007.

28. Browman GP, Wong G, Hodson I, Sathya J, Russell R, McAlpine L, Skingley P and Levine MN: Influence of cigarette smoking on the efficacy of radiation therapy in head and neck cancer. N Engl J Med 328: 159-163, 1993.

29. Chen AM, Chen LM, Vaughan A, Sreeraman R, Farwell DG, Luu Q, Lau DH, Stuart K, Purdy JA and Vijayakumar S: Tobacco smoking during radiation therapy for head-and-neck cancer is associated with unfavorable outcome. Int J Radiat Oncol Biol Phys 79: 414-419, 2011.

30. Meyer F, Bairati I, Fortin A, Gélinas M, Nabid A, Brochet F and Têtu B: Interaction between antioxidant vitamin supplementation and cigarette smoking during radiation therapy in relation to long-term effects on recurrence and mortality: A randomized trial among head and neck cancer patients. Int J Cancer 122: $1679-1683,2008$.

31. Jainkittivong A, Swasdison S, Thangpisityotin M and Langlais RP: Oral squamous cell carcinoma: A clinicopathological study of 342 Thai cases. J Contemp Dent Pract 10: E033-E040, 2009.
32. Sano D and Myers JN: Metastasis of squamous cell carcinoma of the oral tongue. Cancer Metastasis Rev 26: 645-662, 2007.

33. Choi S and Myers JN: Molecular pathogenesis of oral squamous cell carcinoma: Implications for therapy. J Dent Res 87: 14-32, 2008.

34. Chen SF, Yang SF, Li JW, Nieh PC, Lin SY, Fu E, Bai CY, Jin JS, Lin CY and Nieh S: Expression of fascin in oral and oropharyngeal squamous cell carcinomas has prognostic significance-a tissue microarray study of 129 cases. Histopathology 51: 173-183, 2007.

35. Chien MH, Ying TH, Hsieh YH, Lin CH, Shih CH, Wei LH and Yang SF: Tumor-associated carbonic anhydrase XII is linked to the growth of primary oral squamous cell carcinoma and its poor prognosis. Oral Oncol 48: 417-423, 2012.

36. Kang CJ, Lin CY, Wang HM, Fan KH, Ng SH, Lee LY, Chen IH, Huang SF, Liao CT and Yen TC: The number of pathologically positive lymph nodes and pathological tumor depth predicts prognosis in patients with poorly differentiated squamous cell carcinoma of the oral cavity. Int $\mathbf{J}$ Radiat Oncol Biol Phys 81: e223-e230, 2011.

37. Mandal M, Myers JN, Lippman SM, Johnson FM, Williams MD, Rayala S, Ohshiro K, Rosenthal DI, Weber RS, Gallick GE and El-Naggar AK: Epithelial to mesenchymal transition in head and neck squamous carcinoma: Association of Src activation with E-cadherin down-regulation, vimentin expression, and aggressive tumor features. Cancer 112: 2088-2100, 2008.

38. Chen TC, Hsu CW, Lou PJ, Ko JY, Yang TL, Chen CN, Chang YL and Wang CP: The clinical predictive factors for subsequent distant metastasis in patients with locoregionally advanced oral squamous cell carcinoma. Oral Oncol 49: 367-373, 2013. 